\title{
APPLICATIONS OF SPECTROGRAPHY TO ANALYTICAL AND INDUSTRIAL CHEMISTRY.
}

\author{
By S. JUDD LEWIS, D.Sc. (Tübingen), B.Sc. (London), F.I.C.
}

(Read at the Meeting, December 2, 1914.)

Many discoveries of fundamental significance have been made by means of spectrography, and yet the spectrograph is seldom applied to the ordinary purposes of analysis and industry. No doubt this is to be explained by the expensive character of the equipment, and the somewhat extensive practice which is necessary to obtain results which are useful and reliable; also by the limited applicability of any individual outfit.

The most generally useful and convenient apparatus is a spectrograph constructed with quartz prisms and lenses. The dispersion is not great, so that it is not to be preferred for visual work; but the quartz refracts, and allows to pass the whole of the ultra-violet rays from those having a wave-length of about 2,000 Ångström units, as well as those of the visible spectrum. Inasmuch as the more refrangible portion is invisible, its study is of necessity conducted through the medium of photography.

There are four important advantages in this arrangement: First, the photograph is a true and permanent record of the experiment. Secondly, the ultra-violet region is included, where lines are exhibited by several elements which show no lines, or only a few, in the visible region-for example, boron, phosphorus, silicon, antimony, iron, magnesium. Thirdly, most of the absorption spectra studied with reference to chemical constitution have been produced in the ultra-violet. Fourthly, most of the purposes to be served with a visual glass apparatus can be equally well attained with this.

Spectra may be classified primarily into (a) emission spectra, produced from the light emitted by the incandescence of the body under examination, consequently they are usually possible with inorganic matter only; and (b) absorption spectra, obtained by analysing the light which has passed through the substance, usually in solution, which is being investigated. The light in selected parts of the spectrum is absorbed wholly or partially in a manner peculiar to the substance effecting it. The method is available for both organic and inorganic compounds.

Of emission spectra, the flame spectrum is of very limited value. The arc spectrum is the most generally useful, and is available for all metallic and certain non-metallic elements ; with this the spark spectrum is an equal rival for utility, and for solutions almost indispensable. It is usual for spectra, either "arc " or "spark," to be composed of the spectrum of the substance composing the electrodes and the spectrum of the substance under examination.

Whatever may be done in academic research, where there are few restrictions as to time and expense, it may be accepted as a general rule, though not a universal one, that in the practical applications of spectrography only comparison spectra, or spectra photographed on similar principles, call for consideration. The spectra to be 
compared are photographed in juxtaposition, an arrangement which enables the strictest comparison to be made.

A very important application of the method is the study of those mineral constituents of water which occur in traces only, for they must have marked influence on the life-history of both animals and vegetables. For example, the wide distribution of boron in these originates, no doubt, in the natural water-supplies (see "Year-Book of Pharmacy," 1914, 362, where the application of spectrography to drug analysis is also referred to). Minerals, ores, clays, building materials, alloys, impure metals, and the like, are equally ready to reveal their constituent elements.

To summarise, emission spectra can find application in analytical and industrial practice as follows :

1. There is certainty as to the identification of the elements. There can be no doubt as to the presence of a given element, even if only one characteristic group of lines is evident, or even a single line in some circumstances.

2. Substances, the composition of which is entirely unknown, which are difficult to resolve by reason of complexity, insolubility, or otherwise, are analysed completely and without difficulty.

3. Unsuspected elements are readily discovered, for such elements register their spectra on the plate.

4. Very small quantities, whether absolute or relative, are sufficient for disclosing the presence of the various elements composing the material.

5. Those impurities which are difficult of detection by chemical means often reveal themselves at once in the spectrum, as in the case of a trace of barium in a strontium salt "guaranteed free from baryta."

6. Differences in qualitative composition between two similar specimens can be found readily, and identified without the necessity of ascertaining the general composition of either.

7. The qualitative analysis of an inorganic substance with reference to its metallic and certain non-metallic elements is complete.

8. It is rarely necessary to prepare the material in any way for the analysis.

It is apparent that many useful purposes may be served by the more extensive study of spectroscopy, and that the spectrograph can no longer be regarded merely as a means to the ends of academic science, but also as an instrument for investigating and solving many of the practical problems of everyday life.

My thanks are due to Messrs. Adam Hilger, Ltd., for exhibiting a spectrograph with its accessories at the meeting.

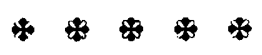

